\title{
Performance Investigation of Channel Noise Effect in Data Transmission Medium Using Signal to Noise Ratio (SNR)
}

\author{
Gebremedhn W. Wagaye \\ School of Electrical and Computer Engineering, Ethiopian Institute of Technology - Mekelle (Eit-M), \\ Mekelle University, Mekelle, Ethiopia Tel: +251914728861/+251911020005
}

\begin{tabular}{|c|c|}
\hline Article Info & ABSTRACT \\
\hline Article history: & \multirow{5}{*}{$\begin{array}{l}\text { The noise introduced in the channel obviously affects the bit error rate of the } \\
\text { communication system and this has direct impact in the security. Here the } \\
\text { main problem is that the receiver terminal decoding techniques can lead to } \\
\text { wrong interpretation even if the Bit Error Rate (BER) is acceptable. So the } \\
\text { main idea here is to introduce high values of Signal to Noise Ratio (SNR) } \\
\text { that can improve the bit error rate which exists due to the noise introduced in } \\
\text { the wireless channel. }\end{array}$} \\
\hline Received Nov 14, 2017 & \\
\hline Revised Jan 8, 2018 & \\
\hline Accepted Apr 21, 2018 & \\
\hline & \\
\hline
\end{tabular}

SNR

BER

Noise

Data and Channel

Copyright (C) 2018 Institute of Advanced Engineering and Science. All rights reserved.

\section{Corresponding Author:}

Gebremedhn W. Wagaye,

School of Electrical and Computer Engineering,

Ethiopian Institute of Technology - Mekelle (Eit-M), Mekelle University, Mekelle, Ethiopia.

Tel:+251914728861/+251911020005

Email: wubet@mu.edu.et, wubetger@gmail.com

\section{INTRODUCTION}

\subsection{Background}

The main objective of the research is to investigate the performance of the communication media or channel interference on information security systems. Therefore, the performance of information or data transmitting through the existing medium is detecting the values of mean square error (MSE) or BER of the SNR [1] at the terminal of the receiver device. In most of case of common channels or mediums, SNR signals are evaluated or investigated in different models. Every channel or medium describes as a distance between sender and receiver with the factors of the channel gain and path loss. So that, channel gain, to model a time variant parameter with several probability distribution functions (PDF). So, investigating the influence of noise on systems performance, different channels are modelled like Additive White Gaussian Noise (AWGN), Rayleigh [2] and Rican [3] and majority we use them in wireless application. So that, received signals are decoding and detecting with different techniques in the receiver end. Such as binary convolution codding, modulation and demodulation and data rating [1].

According to IEEE 802.11 standard [3]. A signal which passes through the AWGN is added a white Gaussian noise to the transmitted data. Additive white Gaussian is the basic channel noise model. A simple additive channel noise disturbs the quality of the transmitted signal or message.

Rayleigh channel model is ideal in multipath reception [2,4] and it is majorly demonstrated for radio signal propagation effect and more applicable for troposphere and ionospheres propagation of signals between receivers and transmitters [5]. Rican channel model is related as Rayleigh channel model [2] except its strong dominant component of the Rican fading channel is usually known as line of sight component [4]. 
AWGN is the most basic and simplest channel model in local positioning and wireless communication system or proximity detector based [6]. When information or data travels over AWGN channel frequently, the Additive White Gaussian noise interference of the channel affects the quality of the signal. Therefore, AWGN is the standard channel models.

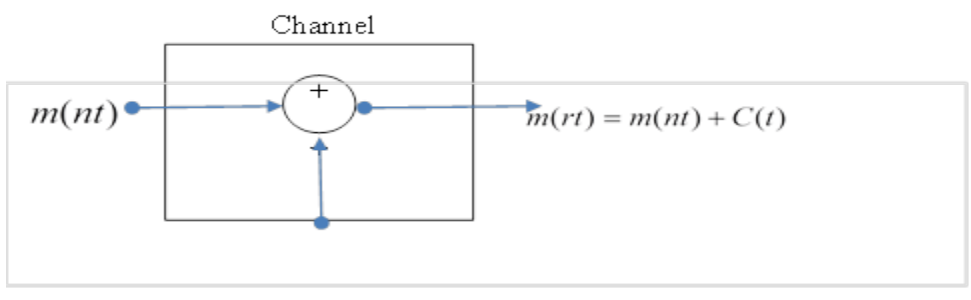

Figure 1. Block diagram of channel model [1]

Where: $m(n t)$ is the transmitted signal, $m(r t)=m(n t)+C(t)$ is the received signal and $C(t)$ is the noise signal.

Bit Error Rate (BER): is calculated as in (1)

$$
\mathrm{BER}=\frac{\text { Bits recieved in error }}{\text { number of bits transmitted }}
$$

In digital or analog transmission medium, the channel noise altered the received bits to increase the bit errors of the information traveling through the transmission medium. SNR is the received to noise signal ratio and it is very important tool in the physical layer of the communication media to measure the quality of received signal or data. In general, noise introduces in the transmission channel includes internal and external noise of the environment and unwanted signals of the systems. Due of those noise strength increasing the processing time and loss of signal increase that leads to BER increases and SNR decreases [3, 4, 6], so to determine the relation of BER or MSE and SNR or PSNR is somewhat difficult in multi-channel environment. SNR is a parameter usually used to calculate the quality of a signal [7] in the communication media and stated in decibels as in (2).

$$
\mathrm{SNR}=10 \log 10\left(\frac{\text { signal power }}{\text { Noise power }}\right) d B
$$

$E b / N o$ is the energy to power spectral noise density ratio [8] and useful to analysis the performance of BER in different digital or analog modulation and demodulation in the data transmission without considering bandwidth.

\subsection{Problem of statement}

The current data transmission system only focus on concealing strategy of the information but not considering the communication channel properties which directly affects the quality of the received signal. Therefore, noise introducing in the transmission medium affects the BER of the data, this has direct impact on the data security and decoding techniques that leads to wrong interpretation. So, by introducing SNR mechanism at the transceiver end we can remove the channel noise effect or BER in the sent signal.

\subsection{Significance of the work}

The proposed of the system is used to solve the channel interference effect in data transmission system, which leads to wrong interpretation at the receiver end, by increasing SNR to improve the BER exists due to the noise introduced in the transmission medium.

\section{SYSTEM MODELING}

Wireless communication channels are an important part of communication systems. Then, a signal or information must be or close to free from error so that, it will have a high or strong signal quality. Here is the core issue to develop a model of the channel effect within the communication system. While there are different ways of modeling the channel, the most basic model of channel noise is AWGN. 


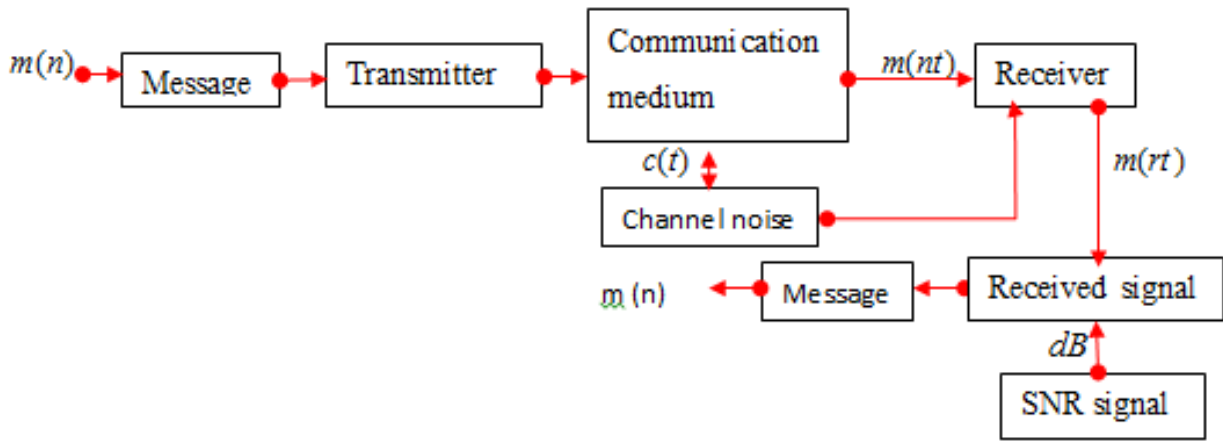

Figure 2. System model

Communication channels are affecting the information pass through it. The signal quality at the receiver side is distorted signal mean that if it is an image the received image is distracted image.

$$
m(r t)=m(n t)+c(t)
$$

Where $m(r t)$ received signal plus channel noise $c(t)$ with Gaussian probability distribution stated as in (4)

$$
c(t, \mu, \sigma)=\frac{1}{\sigma \sqrt{2 \pi}} e^{-\frac{(t-\mu)}{2 \sigma^{2}}}
$$

Where the parameter $\mu$ is the mean value, $\sigma$ is standard deviation and variance is $\sigma^{2}$ [9] To evaluate the peak signal noise ratio of the channel is as Equation (5).

$$
\text { PSNR }=10 \log _{10}\left(\frac{s q r(\max )}{M S E}\right)
$$

Where $C_{\max }$ the maximum pixel value of the data and MSE is defined as:

$$
M S E=\frac{1}{M N} \sum_{x \& y=1}^{M \& N}(S x y-C x y)
$$

Where $\mathrm{x}$ and $\mathrm{y}$ are wave or information coordinates and M\&N are dimension of the medium. Cxy and Sxy are noise signal and transmitted signal respectively

\section{RESULT AND DISCUSSION}

\subsection{Performance analysis of SNR vs. channel noise effects}

The pixel data values of the received information through channel in comparison to the pixel data values of the message sent from the sender are shown in Figure 3. The effect of the noise is shown for different signal to noise ratio (SNR) values.

Comparing the pixels values of the sent data and the received data as shown in Figure 4 affects with noise. The effect of the noise is shown for different SNR values. Therefore, as in equation (5) explain high quality of the received signal as the SNR increases. The sent signal compared to the received signal is aligned or the same because of the SNR value added to remove the noise. 


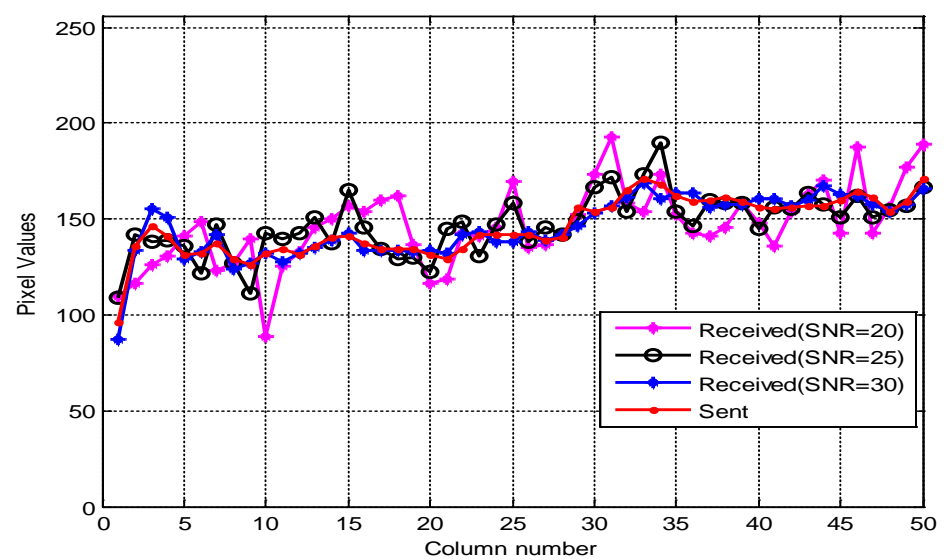

Figure 3. Received and sent pixel data values for different SNR values

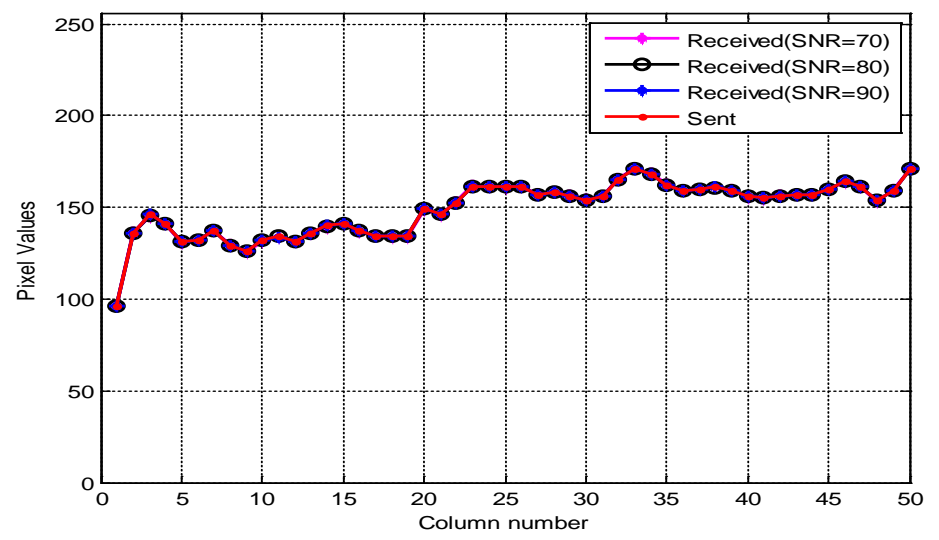

Figure 4. Received and sent pixel values for different SNR values

\section{EVALUATION OF SNR TECHNIQUES}

The retrieving process at the receiver end, the information sent and received represents the data matrix generated before and after transmitting through the media respectively. For SNR=20dB and $\mathrm{SNR}=30 \mathrm{~dB}$ as shown in Figure 3 is used to generate the respective character using the SNR values. Here as in Figure 5, the tolerance value of the SNR for an error $1 / 200$ in the matrix vale introduced to get the original message without distorted. Therefore, the sent and received information are aligned at the same level to show the quality of the signal.

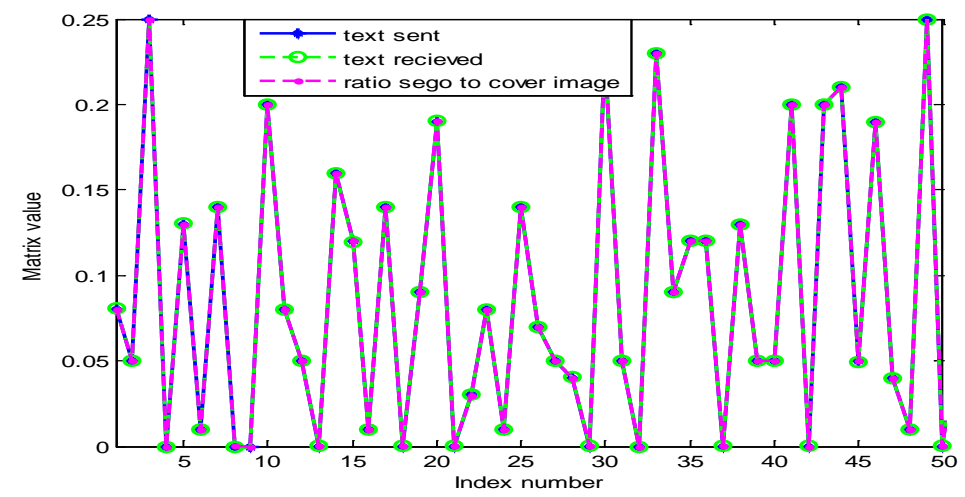

Figure 5. Comparis on of the ratio of received and sent matrix 


\section{CONCLUSION}

Therefore, the quality of the transmitted information depends on the channel noise and pixel values of data to be sent in the communication media. Due to the noise of channel, the SNR will be affected and the bit error rate increases. So as, by SNR values increasing with properties of the media, the channel effect will be decreased and MSE is decreased then will get the quality signal at the receiver end. In really, the processing time will be related with SNR. So if the signal quality is increased the processing time will be increased. Consequently, the process is fast.

\section{REFERENCE}

[1] Deepak K. Chy, Md. Khaliluzzaman, 'Evaluation of SNR for AWGN, Rayleigh and Rician Fading Channels Under DPSK Modulation Scheme with Constant BER', International Journal of Wireless Communications and Mobile Computing. Vol. 3, No. 1, pp. 7-12, 2014.

[2] Amit Grover and Neeti Grover, 'On limits of Wireless Communications in a Fading Environment: a General Parameterization Quantifying Performance in Fading Channel', Indonesian Journal of Electrical Engineering and Informatics (IJEEI), Vol. 2, No. 3, pp. 125 -131, September 2014.

[3] IEEE standard for wireless LAN: Medium Access Control and Physical Layer Specification, P802.11, January 1999.

[4] A. Alimohammad, S.F.Fard, B.F.Cockburn and C.Schlegal, "Compact Rayleigh and Rician fading simulation based on random walk processes”, IET Communications, Vol.3, No. 8, PP.1333-1342, 2009.

[5] Yahong Rosa Zheng and Chengshan Xiao, "Simulation models with correct statistical properties for Rayleigh fading channels”, IEEE Transactions on communications, Vol. 51, No. 6, June 2003.

[6] Fumiyaki Adachi, "error Rate Analysis of Differentially Encoded and detected 16-APSK under Rician fading”, IEEE Transactions on Vehicular Technology, Vol. 45, No.1, February 199

[7] C. S. Preetham, M. S. G. Prasad, D. S. S. L. Saranya, Charan Teja Somepalli, D. Bhargava Satya Sai Krishna, V. Rohit 'Performance Analysis of Cooperative Hybrid Cognitive Radio Network with Various Diversity Techniques', International Journal of Electrical and Computer Engineering (IJECE), Vol. 6, No. 5, pp. 2125-2133, October 2016.

[8] Iskandar and D. Hidayat, 'SC-FDMA LTE Performance through High Altitude Platforms Communications (HAPS) Channel', TELKOMNIKA (Telecommunication, Computing, Electronics and Control), Vol.14, No.2, pp. 515-522, June 2016.

[9] BoWen Wang and Quan Gu 'A Detection Method for Transmission Line Insulators Based on an Improved FCM Algorithm', TELKOMNIKA (Telecommunication, Computing, Electronics and Control), Vol.13, No.1, pp. 164 -172, March 2015. 